\title{
LXVI. An account of some electro-magnetic combinations, for exhibiting thermo-electric phænomena, invented by Mr. James Marsh of Woolwich: with experiments on the same
}

\section{Peter Barlow Esq. F.R.S.}

To cite this article: Peter Barlow Esq. F.R.S. (1823) LXVI. An account of some electro-magnetic combinations, for exhibiting thermo-electric phænomena, invented by Mr. James Marsh of Woolwich: with experiments on the same , Philosophical Magazine Series 1, 62:307, 321-327, DOI: $10.1080 / 14786442308644416$

To link to this article: http://dx.doi.org/10.1080/14786442308644416

曲 Published online: 29 Jul 2009.

Submit your article to this journal ¿

Џ Article views: 3

Q View related articles ¿ 


\title{
PHILOSOPHICAL MAGAZINE AND JOURNAL.
}

\author{
$30^{\mathrm{th}} \quad N O V E M B E R \quad 1823$.
}

LXVI. An Account of some Electro-magnetic Combinations, for exhibiting Thermo-electric Plunomena, invented by $M r$.

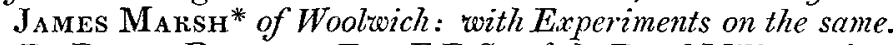
By Peter Barcow, Esq. F.R.S., of the Royal Military Academy, Honorary Member of the Cambridge Philosophical Society and of the Society of Civil Engineerst.

DROFESSOR CUMMING having very obligingly favoured me with a copy of his highly interesting paper printed in the Transactions of the Philosophical Society of Cambridge, for the present year, ' On thermo-electric Phanomena,' I put it into the hands Mr. Marsh, requesting him to copy any of the apparatus he there might find described, with a view to repeating such of the experiments as were reported. This he very readily undertook, and soon brought me not only those I had requested him to copy, but several new ones, which latter it is my intention to describe in this paper. The most essential apparatus in these experiments is a very sensible galvanometer. Professor Cumming, who was the first to employ this useful machinet, has not very distinctly stated his construction of it. I cannot tell, therefore, how nearly that I am about to describe may resemble his; at all events Mr. Marsh's galvanometer is a very simple and sensible instrument, and its construction will not be uninteresting to some of your readers.

$\mathrm{AB}, \mathrm{CD},(\mathrm{Pl}$. V.) fig. 1, are two wooden supports or pillars, through which pass brass wires, having each at its extremity a small brass cup; to the other extremity of each is attached by good contact the square helix cage shown in the figure: on the top of the prop $\mathrm{E}$, is a fine point carrying a very light and delicate compass needle with a card below; FG is a stand for holding the bar RS, of bismuth, antimony, or other metal; and $n m n^{\prime} m^{\prime}$, are wires of a different metal solcered or bound round at the ends $R$ and $S$. The set screw at $s$ is for adjusting to any height. The brass cups being now

* We are glad to find that this ingenious self-taught artist has commenced the business of chemical and philosophical instrument-maker in Woolwich.--Enit.

+ Communicated by the Author.

I See Trans. Camb. Phil. Socicty for 1821 .

Vol. 62. No. 307. Nor, 1823. 

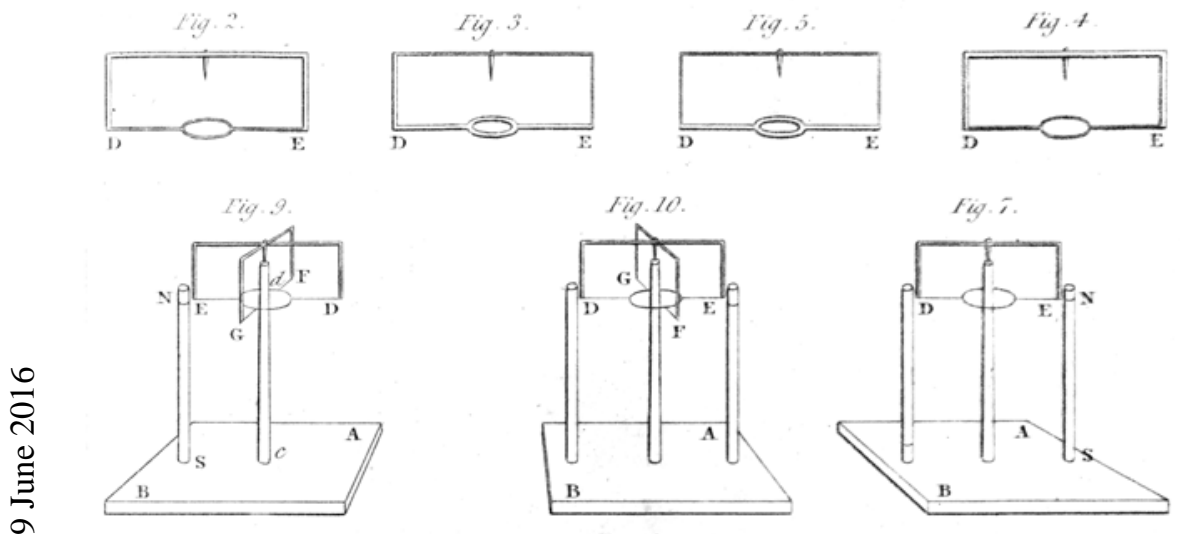

Fig. - .

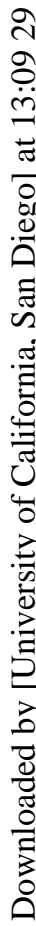
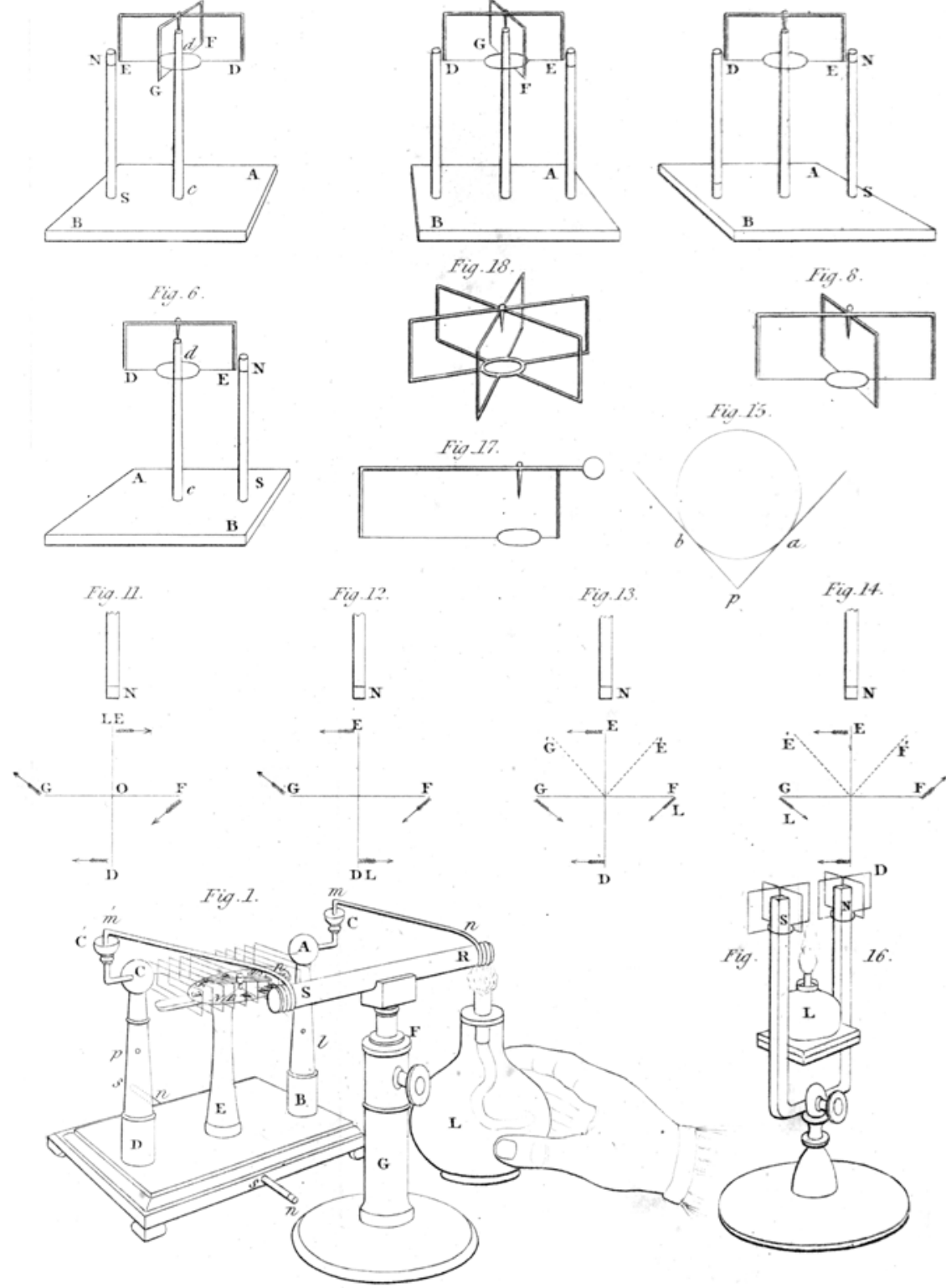
rubbed with a little nitrate of mercury, and pure mercury being poured into them, the contact is made or broken at pleasure, by placing the wires in or throwing them out of the cups; and the effect thus produced is shown in the most sensible manner by the needle within the helix; which in very delicate cases may be neutralized by the small manets $n s$, ns in the foot below, when the instrument is placed east and west, or by inserting them in holes, for the purpose, in the props $A B, C D$, as seen at $p$ and $q$ when it is in the meridian. Then by applying the lamp at $R$, then at $S$, changing the bars and wires, and the size of the latter, \&c. all the variety of experiments so judiciously arranged and combined by Professor Cumming may be repeated, and the series extended at pleasure. The galvanometer of Professor Cumming is undoubtedly the same in principle as the above, and may not perhaps differ much in construction; but as he has not described it, the foregoing description will not, I hope, be thought superfluous *.

\section{Apparatus for exhibiting Rotation.}

Professor Cumming at the conclusion of his paper has suggested a small combination of platinum and silver wires for exhibiting a rotation about a magnet, as Mr. Faraday had done in the case of a galvanic wire, in his experiments on electro-magnetism, and which certainly threw more light on the inquiry than any experiments before made on the subject.

On constructing a machine precisely from the description given by Professor C., it was found that it had indeed a tendency to revolve, but so small that it was very difficult, if not impossible, to exhibit the phænomenon in a satisfactory manner. It turned out, however, while carrying on the experinent, that, although a magnet in the interior of the wire would not produce any but a weak tendency to rotation, a magnet applied exterior to it was capable of producing the most decided effect, which will be seen as I proceed to describe the following experiments.

For this purpose four rectangles, figures 2, 3, 4, 5, were made of platiuum and silver combined, as shown in the figures, where the thicker lines indicate silver wire, and the lighter ones the platinum; a ring being formed below to admit the prop upon which they were to revolve; and a fine steel point brazed to the upper side to rest in the agate on the top of the prop. The stand with a rectangle suspended is shown in figure 6 , where $A B$ is a board, $c d$ a brass prop with its agate at top, and N S a magnet placed as nearly as possible to the

* Professor Cunming has since described his instrument in the Annals of Plilosophy for October lest.-Eor

wire. 
wire. The spirit-lamp being now applied at $\mathrm{D}$ or $\mathrm{E}$, the rotation will commence, either to the right or left, according to circumstances, and which will be reversed by reversing the pole of the magnet. Fig. 7 shows the same stand with two magnets.

This being premised, the reader will easily follow me in the detail of the following experiments, observing that when the motion is said to be to the right or left, he must imagine himself coinciding in position with the wire about which the machine turns.

Exp. 1. The rectangle, fig. 2, being applied upon the stand, fig. 6 , and the lamp at $\mathrm{E}$, the rectangle was projected to the right till $\mathrm{D}$ reached the lamp*; it was then propelled back again, and after a few oscillations it remained at rest at right angles to its first position.

Exp. 2. The rectangle adjusted as before, and the lamp applied at $\mathrm{D}$, the wire was projected to the left with similar results to the preceding.

Exp. 3. The rectangle adjusted so that $D$ was next the magnet, and the light then applied at $\mathrm{D}$. The wire projected to the right.

Exp. 4. The rectangle still in the same position, but the light applied at the other extremity. The wire was projected to the left.

Exp. 5, 6, 7, 8, were made under precisely the same circumstances with the rectangle, fig. 3 ; and the results were similar, but much weaker, and the motions all reversed.

Exp. 9, 10, 11, 12, were made with the rectangle, fig. 4 . The motions the same as with rectangle, fig. 2, except that we generally obtained a rotation when the light was applied as in Experiments 2 and 4.

Exp. 13, 14, 15, 16, were still the same experiments, but with the rectangle, fig.5. The results were as in the above case, but reversed in respect to direction, and inferior in force.

A similar set of experiments were made with the south pole of the magnet opposed to the wire, and the results were similar, but all in the reverse direction.

As it was obvious from these results that the rectangle, fig. 4, either from its more accurate balance, or from the nature of the combination, was the most powerful, it was alone made use of in the following experiments, in which two magnets were employed.

Exp. 17. The rectangle, fig. 4, being suspended as shown

* It onght rather to be said before $\mathrm{D}$ reached the lamp; for when by chance it did reach it, the wire revolved.

$S: 2$ 
in fig. 7, and the lamp applied at $\mathrm{E}$, a rotation immediately commenced to the right, which soon increased to 30 revolutions per minute.

Exp. 18, The rectangle adjusted as before, and the lamp applied at $\mathrm{D}$, the rotation to the left, at the rate of 30 revolutions per minute.

Exp. 19. We now suspended the compound rectangle, fig. 8, and opposed to it the north end of the magnet, as in fig. 9, and applied the lamp successively at E, D, G and F. The following were the results :

Lamp at $\mathrm{E}$, rapid rotation to the right.

Lamp at $\mathrm{D}$, rotation to the left, 30 per minute.

Lamp at $\mathrm{G}$, rotation to the left, ditto.

Lamp at $\mathrm{F}$, no tendency to rotation.

Exp. 20. The magnet reversed, the lamp applied as before. Lamp at $\mathrm{E}$, rapid rotation to the left.

Lamp at $\mathrm{D}$, rotation to the right, 30 per minute.

Lamp at $G$, no tendency to rotation.

Lamp at $F$, rotation to the right, 30 per minute.

Exp. 21. The same experiments were repeated with two magnets with contrary poles opposed, as shown in fig. 10 .

The results as above, but more rapid: in the last experiments we obtained about 30 revolutions per minute, but it was impossible to count them in these, when two strong magnets were employed.

It should be observed that in no case could any strong tendency to rotation be observed when a magnet was employed for a support, and the exterior magnet removed : fig. 17, in which one branch is carried further from the magnet, showed the greatest tendericy.

A pleasing exhibition of a compound motion of this kind is shown in fig. 16 , in which NS is a horse-shoe magnet with an agate at each pole to rest the wire in, and $L$ a lamp which serves to heat each compound rectangle. 'The motion in this case is not so rapid as in the former, but it is very pleasing, and will continue as long as the lamp burns.

Compound rectangles with six branches (fig. 18) were tried, but there appeared to be little or nothing gained by this increase of number. It appears, I think, that one with four branches performs upon the whole the best. The length of the rectangles is about two inches, the depth an inch; the diameter of the platinum wire $\frac{1}{60}$ th of an inch, and that of the silver $\frac{1}{4} \pi$ th ; but it is by no means necessary to observe these dimensions. In general it may be stated, that the lighter the rectangle, the greater will be its velocity.

Let us now endeavour to trace the theory of these motions. 
It must already have struck the reader as remarkable, that out of the four positions in which the lamp may be applied in the double rectangle (fig. 9), two of them give a rotation in one direction, and one of them in an opposite one, and that at the fourth point there should be no tendency to rotation whatever, but on the contrary a decided direction; this latter point is to the right of the north pole, or to the left of the south pole of the magnet, the observer assuming his position to coincide with the axis of motion, as already suggested in the preceding part of this article. A satisfactory illustration of this singular phænomenon will, it is presumed, be considered a strong test in favour of the hypothesis on which it is founded, particularly if the same hypothesis should be found competent to the ilhustration of every other electro-magnetic phænomenon hitherto observed.

In my "Essay on Magnetic-Attractions and on Electromagnetism," I have shown that, by supposing the galvanic conducting wire to act upon the magnet with a tangential force varying inversely as the square of the distance, we may not only illustrate but compute the effect of any proposed combination; let us then see how far this same supposition wili assist us in explaining the singular anomaly above mentioned.

The platinum being positive to the silver; when the lamp is applied at the union of the two metals, the fluid will be transmitted through the silver wire under the same circumstances as in a galvanic apparatus, with two plates, it is transmitted through the conducting wire from the copper side to the zinc; it ought therefore, when thus transmitted, to project the north pole of the magnet to the left, the observer now coinciding in position with the wire through which the circuit passes. Or the magnet being fixed, and the wire free as in our case, this latter ought to be projected to the right hand. Thus referring to fig. 9 , and conceiving the lamp to be applied at $\mathrm{E}$, the point $\mathrm{E}$ ought to be projected to the right hand of the observer, looking towards the magnet, while the points $\mathrm{F}, \mathrm{D}$, and $\mathrm{G}$ (the circuit in these being descending) ought to be projected to the left, the observer conceiving himself coinciding in position with these respective wires and always looking towards the magnet. On the contrary, when the light is applied at $F$, then the circuit being ascending at $\mathrm{F}$, and descending in all the other branches, this ought to be projected to the right, and all the others to the left; and so on, for any wire to which the lamp is immediately applied: and of course the contrary to all this ought to happen when the south pole is applied. Let figures 11, 12, 13, 14, represent the four applications of the lamp as stated in experiment 19; and the 
several arrows the direction of rotation as excited by the magnet and lamp in these four cases. Then it is obvious that in fig. 11 , answering to the case of the lamp applied at $\mathrm{E}$, the tendencies to rotation are all in one direction, and we ought therefore to expect, as is actually the case, the rotation to be very rapid. In figure 12 , two of the forces are in one direction, and two in the other; and therefore if these forces were all equal, we ought to have no motion. But the force at $D$ is very considerable in comparison with that at $F$ or $G$, both on account of the immediate application of the lamp at $\mathrm{E}$, and the division of the circuit into three branches at $\mathrm{O}$; and the direction of the two latter forces on the respective levers, which being oblique there are necessarily less effective in producing rotation. Again, the force at $E$ is also considerable in consequence of the proximity of the magnet; so that the

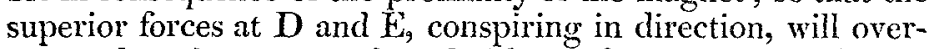
power the other two weak and oblique forces, and produce a very considerable rotation, although inferior to the former.

In fig. 13, the forces at $E$ and $G$ will be the superior forces; and as they conspire in direction, they will overpower the two inferior forces at $\mathrm{D}$ and $\mathrm{F}$, which are opposed to them, and a considerable rotation will again ensue.

In fig. 14, the two superior forces also conspire, but with this peculiarity, that the moment the motion ensues, and the $\operatorname{arm} \mathrm{F}$ arrives at $\mathrm{F}^{\prime}$ and $\mathrm{E}$ at $\mathrm{E}^{\prime}$, the direction of these two forces no longer assists in giving rotation, being then both in the direction of the radii from the centre, and the resultant acts to bring the machine directly towards the magnet, and thereby to convert the rotation into direction, which the experiment strongly exhibits.

When the sonth pole of the magnet is opposed to the wire, all these directions of motion will be reversed, and then of course the point of neutralization will be at $G$, fig. 15: which explains the apparent anomaly of the point of no action being to the right hand of the north pole and to the left of the south pole. In fig. 13 we have seen that with the lamp applied at $G$ the motion is to the left; and when at $E$, as in fig. 11 , it is to the right; it follows, therefore, that between these two there ought to be some position of equilibrium as G', fig. 13, and where no motion of course ought to ensue. But in this case, instead of the forces at $G^{\prime}$ and $E^{\prime}$ being directed from the centre as in the case of fig. 14, they are directed to the centre. So that this state of equilibrium differs from the former in this, - that in the former, the equilibrium is one of stability, and in the latter of instability, and consequently very difficult to exhibit: in fact, the slightest inclination of 
the flame of the lamp in this situation of it, will give rise to a slow rotation in either direction according to the circumstances of the case. The same principles will enable us to explain the increase of acceleration produced by two magnets with their opposite poles applied as in fig. 7 and fig. 10, and the cause of the non-action of a central magnet, except as in the apparatus of Professor Cumming, where one of the branches is carried further from the centre; in which case a slight tendency to rotation is exhibited equal to the difference of the two opposite forces.

We see thus the marked difference between the electromagnetic rotations produced by the application of the lamp, as in the cases above, and those produced by the galvanic machine; in the latter case it is essential to have the magnet central, whereas in this the magnet must be exterior to produce the desired effect; and the reason is obvious (referring for example to Experiment X, Essay on Magnetic Attractions), for here, and in all similar cases, the fluid being transmitted from the centre passes down the several branches in the same direction, and is therefore acted upon by the central magnet all in one sense; whereas in these, the fluid being ascending in one branch, and descending in the other, the forces on one side counterbalance those on the other, and the machine remains quiescent.

It has been objected against the hypothesis I have advanced of a tangential force varying inversely as the square of the distance, that if such were the case, the cylinder alluded to above (in Experiment $X$ of my Essay) ought to revolve by the application of an exterior magnet; whereas it is almost entirely insensible to its action. But this objection must fill, if the nature of the forces on the periphery of a circle from an exterior point be properly considered; for let $p$ (fig. 15) be an exterior point, and $p a, p b$, tangents to the circle $a b d$, then the part of the circumference between $a$ and $b$ will be acted upon in one direction, and all the other part of the circumference in the opposite one; and although the line of action is more considerable in the latter case, the intensity is greater in the former, and the difference between the two is not sufficient to cause the rotation. In other words, the centre of attraction of the periphery of a circle estimated from a point without, falls so near to its centre, that the effect to produce rotation is too weak to render itself sensible.

P.S.-It may be proper to observe, that for the convenience of making the drawings I have represented the magnet as standing upright; but the experiments were generally made with powertul magnots placed horizontally.

LXVII. On 